\title{
Relación universidad-empresa-estado. Un análisis desde las instituciones de educación superior de Barranquilla-Colombia, para el desarrollo de su capacidad de innovación
}

\author{
Karelis del C. Barrios-Hernández* y Enohemit Olivero-Vega \\ Facultad de Administración y Negocios, Universidad Simón Bolívar, Barranquilla-Colombia. \\ (correo-e: kbarrios22@unisimonbolivar.edu.co; karelisbarrios03@gmail.com) \\ * Autor a quien debe dirigirse la correspondencia.
}

Recibido Ago. 2, 2019; Aceptado Oct. 4, 2019; Versión final Dic. 30, 2019, Publicado Abr. 2020

\begin{abstract}
Resumen
Se analiza la relación universidad-empresa-estado, desde las instituciones de educación superior para el desarrollo de capacidad de innovación en Barranquilla, Colombia. Se propuso una revisión de las actividades que involucran relaciones con el sector externo realizadas por este tipo de instituciones. Se planteó una investigación cualitativa apoyada en la revisión documental que resultó valiosa para contrastar los referentes bibliográficos con las acciones realizadas por las instituciones. Los principales hallazgos destacan que acorde al tipo de carácter académico, las universidades son quienes actualmente fortalecen sus procesos de docencia como su misión tradicional, y paralelamente desarrollan actividades de investigación y extensión que contribuyen al desarrollo del país. Como resultado final se encontró que, la triada universidad- empresaestado es la alianza adecuada para el desarrollo de innovaciones que respondan a las necesidades y oportunidades del entorno.
\end{abstract}

Palabras claves: instituciones de educación superior; capacidad de innovación; empresa; estado.

\section{University-enterprise-state relationship. An analysis from higher education institutions of Barranquilla-Colombia, for the development of their innovation capacity}

\begin{abstract}
The university-business-state relationship, from the point of view of the innovation capacity of the higher education institutions in Barranquilla, Colombia is analyzed. A review of the activities that involve relations with the external sector made by this type of institutions was proposed. A qualitative research was carried on based on the documentary review that was valuable to contrast the bibliographic references with the actions carried out by the institutions. The main findings indicate that according to the type of academic character, Universities are the institutions that strengthen their teaching processes as their traditional mission, and also develop research and extension activities that contribute to the development of the country. As a final result, it was found that the triad university-business-state is the appropriate alliance for the development of innovations that respond to the needs and opportunities of the surroundings.
\end{abstract}




\section{INTRODUCCIÓN}

En el contexto actual, caracterizado por un elevado dinamismo del entorno, la construcción de entornos colaborativos que propicien la innovación son una pieza clave para el desarrollo de la competitividad en las regiones. En este sentido, la Universidad, la Empresa y el Estado se articulan de forma conjunta para promover sinergias y el aprovechamiento de sus talentos para el desarrollo de la capacidad de innovación. Esta capacidad se genera a partir de la dinámica de la relación Universidad-Empresa y Estado con el fin de poder acercar a los investigadores a las necesidades latentes del entorno en el que se desenvuelven, de tal manera que los proyectos respondan con soluciones prácticas e innovadoras que sin duda alguna mejoran la competitividad de un país. En este sentido, esta relación es clave para mejorar las condiciones de innovación en una sociedad basada en el conocimiento. Además, es un tema que ha cobrado importancia desde 1980, sobre todo por su papel en el proceso de innovación, evidenciado en numerosos estudios que muestra la relación entre la universidad, el entono y las actividades de investigación y desarrollo (Castellanos et al., 2003; Etzkowitz y Leydesdorff, 2000)

El término capacidad se puede concebir como la construcción de la sabiduría colectiva en cualquier organización (Garzón, 2013), las cuales nacen a lo largo del tiempo mediante las interacciones que surgen entre los recursos de la empresa (Venture, 1994), enraizados en la historia de la organización e implican la integración de experiencias pasadas para la solución de problemas actuales, y la orientación de decisiones futuras que evolucionan en el tiempo mediante un proceso de aprendizaje. Por tanto, el concepto de capacidad organizacional está vinculado a dos términos: acción y desempeño (Dávila, 2013) donde se da el proceso de asignación y combinación de los recursos que ofrecen a la gerencia de la organización alternativas de decisión para producir salidas (Winter, 2003). Es así como, una organización posee una capacidad cuando hace algo que es apreciado, y lo hace de manera efectiva, comparado con otras organizaciones menos capaces de obtener una solución (Dávila, 2013)

En este sentido, la capacidad de innovación es definida por Wang y Ahmed 2007) como la habilidad de la empresa para el desarrollo de nuevos productos y servicios, el desarrollo de nuevos métodos de producción, la identificación de nuevos mercados, el descubrimiento de nuevas fuentes de suministro y el desarrollo de nuevas formas organizativas. Esta definición guarda una estrecha relación con Burns y Stalker (1961) quienes la definen como la capacidad de adoptar o poner en prácticas nuevas ideas, procesos o productos con éxito y por Kogut y Zander (1992) como la capacidad para movilizar los conocimientos de sus empleados y combinarlos para crear nuevos conocimientos que derivan en productos o procesos de innovación. En definitiva, la capacidad de innovación se refiere a la habilidad de una organización de transformarse permanentemente con el fin de responder o adaptarse a entornos dinámicos. Por tanto, es el resultado de un proceso de acumulación de conocimiento, que puede verse afectado por condiciones facilitadores e inhibidoras de dicha capacidad (Cohen y Levinthal, 1990). Para Garzón (2013), la capacidad de innovación incluye a toda la organización, porque se logra en su conjunto que las organizaciones, vuelvan explicito el conocimiento individual, lo acumulen, codifiquen y almacenen en memorias organizacionales y patentes para el uso colectivo presente y futuro. De esta manera, la capacidad de innovación se configura como un elemento clave para la obtención de resultados empresariales a través de los procesos de explotación, exploración y ambidestreza organizacional (Acosta y Fischer, 2013).

Es así como la innovación como capacidad organizacional permite un equilibrio entre las actividades de exploración y explotación de recursos y capacidades (Vivas, 2013), ya que una empresa puede generar muchas innovaciones de una pequeña cantidad de nuevos conocimientos o, por el contrario, puede carecer de la capacidad para explotar una gran base de conocimientos que ha generado y mantenido (Lane et al., 2006). Esta acción implica necesariamente el conocimiento y la relación con el entorno para responder a problemas reales. En los países desarrollados, son frecuentes las innovaciones generadas en los parques tecnológicos, donde empresas, universidades y otras organizaciones trabajan colaborativamente (Casallas et al., 2011; Morales et al., 2012; Barrios-Hernández, et al., 2017), con el propósito de impactar de manera positiva en la sociedad. Partiendo de la importancia de la relación Universidad-Empresa- Estado, este articulo pretende contribuir al enriquecimiento de la literatura a través de dos enfoques, en primer lugar, se avanza en el constructo capacidades dinámicas y en segundo se analizan los productos resultantes a partir del relacionamiento de las Universidades.

\section{OTROS ANTECEDENTES}

En la medida que las organizaciones se adaptan y responden a las condiciones cambiantes del mercado a través del desarrollo de innovación, establecen contacto efectivo con los actores del entorno mejorando la posición competitiva de los países. Es allí donde la relación Universidad- Empresa- Estado juega un papel fundamental para la generación de proyectos de gran impacto en las regiones, los cuales, alineados a la estrategia y política nacional de ciencia, tecnología e innovación, integren el conocimiento al desarrollo social, económico y cultural del país. 
La evolución de esta relación, inicia con la incorporación de la investigación como segunda misión de la universidad después de la enseñanza (Bueno, 2007). Posteriormente, la demanda de la sociedad hacia la existencia de universidades con una mayor participación en desarrollo del territorio, propició la evolución de este tipo de instituciones, hacia lo que se ha denominado la tercera misión (Bueno, 2017; Blanco-Ariza y otros, 2017). Esta misión es complementaria a las actividades de docencia e investigación, debido a que a través de ella la Universidad se relaciona con su entorno, es decir, con la sociedad, las empresas y el estado (Barrios \& Olivero, 2015). Por tanto, como función de extensión de las IES abarca todas aquellas actividades relacionadas con la generación, uso, aplicación y explotación, fuera del ámbito académico, del conocimiento y de otras capacidades de las que disponen las universidades (Blanco-Ariza y otros, 2017). De esta manera, la tercera misión asume un rol protagónico, al fomentar y fortalecer los vínculos entre la universidad y el entorno socioeconómico, porque desde la perspectiva universitaria, uno de los ejes principales de cualquier IES es fortalecer y consolidar los procesos de investigación básica y aplicada en las diferentes unidades académicas, así como lograr la transversalidad en el currículo, por lo que se hace necesario establecer relaciones con el sector productivo, el estado y la sociedad.

Es así como el desafío del sistema universitario actualmente es responder a las necesidades del entorno a través de sus procesos misionales de docencia, investigación y extensión. No obstante, la mayoría de las IES no se ha planteado la innovación como algo propio del sistema, pensando que esta es una actividad exclusiva del sistema empresarial. La capacidad para generar innovación tiene mucho que ver con la posibilidad de trabajar interdisciplinarmente, que supone elementos de hibridación. Sin embargo, los entornos académicos son muy cerrados y no resulta fácil desarrollar innovación (Gros y Pablo, 2009).

De acuerdo con Villaveces (2006) las primeras relaciones entre la academia y el entorno se representan en el modelo lineal o modo I, bajo un esquema de oferta- demanda de transferencia del conocimiento. Posteriormente, el modo 2, dio paso a modelos más concretos de relación entre universidades, empresas y Estado. Por una parte, el modelo del triángulo de Sábato y Botana (1968). En la figura 1. Se aprecia que este modelo de política científico-tecnológica, sus vértices son el Gobierno, el sector productivo y la infraestructura científico- tecnológica, quienes están relacionados fuertemente. El primero con la finalidad de formular políticas y movilizar recursos desde y hacia los otros vértices, el segundo provee bienes y servicios y el ultimo contiene a las instituciones de investigación científica y tecnológica.

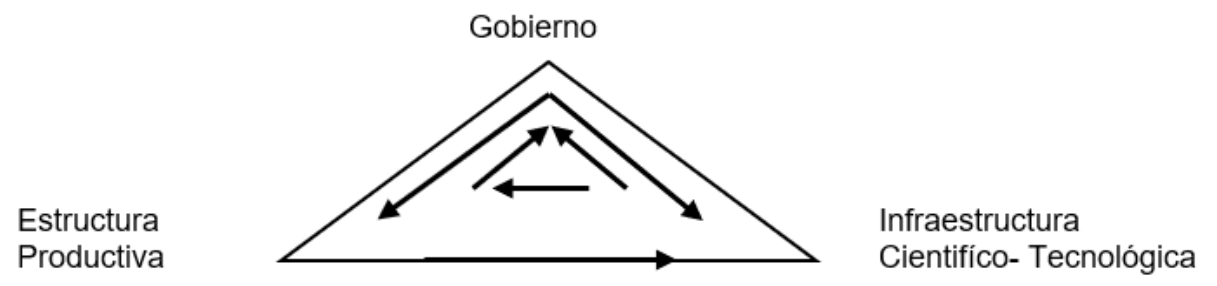

Fig. 1. Modelo de triangulo de Sábato

Por otro lado, el modelo de sistemas de innovación (Lundvall, 1997), tal como se evidencia en la figura 2. Este modelo propone que el estado abarca a la industria y la academia, y regula sus relaciones. La Triple Hélice I es vista como un modelo de desarrollo que ha fracasado, debido a que la innovación fue desalentada en lugar de fomentarse.

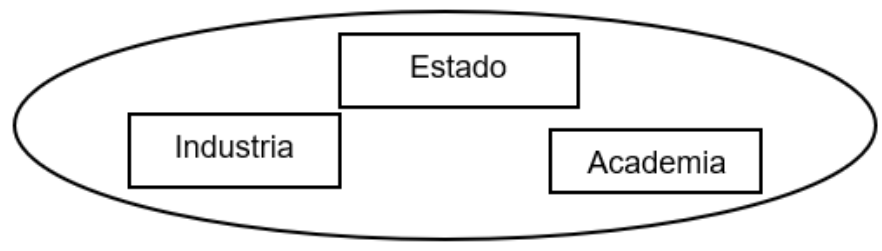

Fig. 2. Modelo de sistemas de innovación

Finalmente, el de la triple hélice (Etzkowitz y Leydesdorff, 1995), expuesto en la figura 3., en su versión II, también recibe el nombre de modelo de laissez -faire de la Universidad- Empresa y el Gobierno. Aquí se da una separación de las esferas institucionales y circunscripción de las relaciones entre ellas. Mientras que en la figura 4., se evidencia que la versión triple hélice III, superpone las esferas institucionales, de manera que de estos espacios de interfaz emergen organizaciones hibridas. Las relaciones de la Triple Hélice son un componente clave en la estrategia de la innovación tanto a nivel nacional como multinacional. 


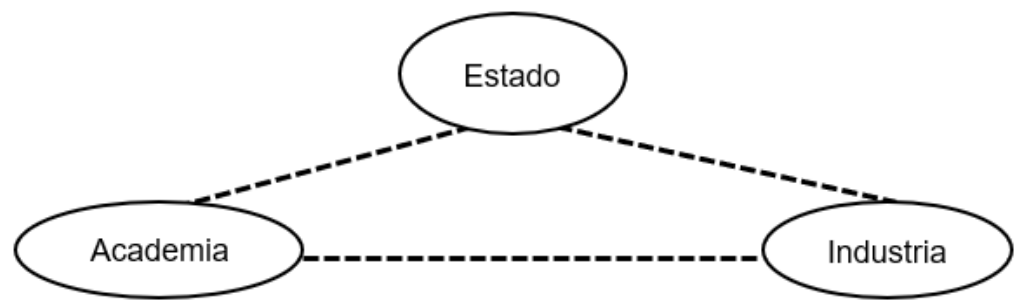

Fig. 3. Modelo triple hélice versión II

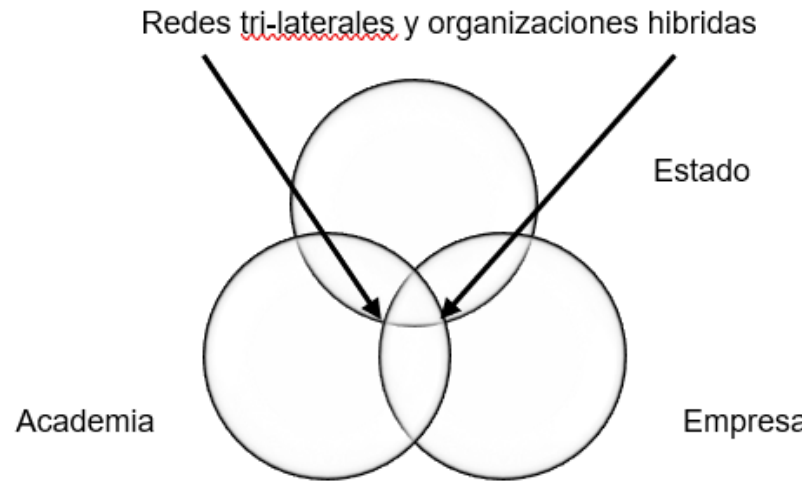

Generador de una infraestructura de conocimiento en función de la interrelación de las

Fig. 4. Modelo triple hélice versión III

En definitiva, los diferentes modelos, han demostrado que, para el desarrollo de la innovación, se requiere de la construcción de entornos colaborativos. Lo anterior indica que el desarrollo de la capacidad de innovación en las IES, depende de tres factores claves: la relación entre el exterior y el interior de la empresa, la relación entre las subunidades dentro de la empresa, y la relación entre los individuos dentro de cada subunidad (Cohen y Levinthal, 1990). Por tanto, para que la relación Universidad- Empresa- Estado, contribuya al desarrollo de innovación, se deben establecer alianzas y convenios entre las Universidades, con el sector productivo y con el gobierno. Por tanto, para garantizar el éxito de los entornos de colaboración, se deben establecer relaciones efectivas que permitan la transferencia no solo de conocimiento, sino el resultado práctico a través del desarrollo de innovaciones que satisfagan necesidades o aprovechen oportunidades actuales del entorno. En este sentido, en la medida que las organizaciones involucradas tengan claridad acerca de su propia estrategia y conciban la colaboración como algo más que un instrumento para alcanzar fines y conseguir ventajas individuales u objetivos en el corto plazo, los esfuerzos compartidos les permitirán alcanzar metas a largo plazo (Roncancio, 2011).

\section{METODOLOGÍA}

Para la contratación de las teorías consultadas se realizó un estudio exploratorio en las IES de carácter privada, ubicadas en Barranquilla-Colombia registradas en el Sistema Nacional de Información de Educación Superior (SNIES), de las cuales seis corresponden a universidades que se identificarán con la inicial de U, seis a instituciones universitarias cuya identificación en esta articulo será de IU y una institución tecnológica identificada con IT. Sin embargo, se trató esta variable como ceteris paribus, concentrando la atención en los elementos que convergen.

Teniendo en cuenta la población seleccionada para la realización de la investigación, se consideró apropiado realizar una investigación cualitativa. Se optó por este enfoque teniendo en cuenta que facilita acercamientos a la realidad de fenómenos y eventos que pueden ser contrastados con referentes teóricos (Talaya y Collado, 2014). Asimismo, la revisión documental sirvió como base para analizar la relación Universidad- EmpresaEstado. Las fuentes secundarias consultadas en su mayoría fueron artículos científicos y documentos de bases de datos como Scielo, Scopus, Elseiver y Emerald. En este sentido, en la revisión de la literatura, la primera línea de trabajo fue con respecto a la búsqueda de información sobre los procesos de investigación y de extensión de Instituciones de Educación Superior de Barranquilla. Los resultados en investigación se analizaron; por un lado, a través del informe de medición de grupos de investigación y el reconocimiento de investigadores del Sistema Nacional de Ciencia, Tecnología e Innovación realizado por Colciencias. Adicionalmente, se revisaron los documentos institucionales publicados en las páginas web de las IES con el fin de comprender como desarrollan sus actividades con el sector externo. Finalmente, en la segunda línea de trabajo se contrastaron la información revisada con los aportes y reflexiones de la literatura con base en las experiencias y observaciones que se tienen de la relación de Universidad- Empresa- Estado a través de casos de éxito identificados. 


\section{RESULTADOS}

Los resultados en investigación de las Instituciones de Educación Superior se analizaron a través del informe de medición de grupos de investigación y el reconocimiento de investigadores del Sistema Nacional de Ciencia, Tecnología e Innovación realizado por Colciencias en el año 2017. En la figura 5. Categorización de grupos de investigación se puede observar como son las IES con carácter académico de Universidades las que presentan grupos de investigación con la máxima categoría de A1 y A.

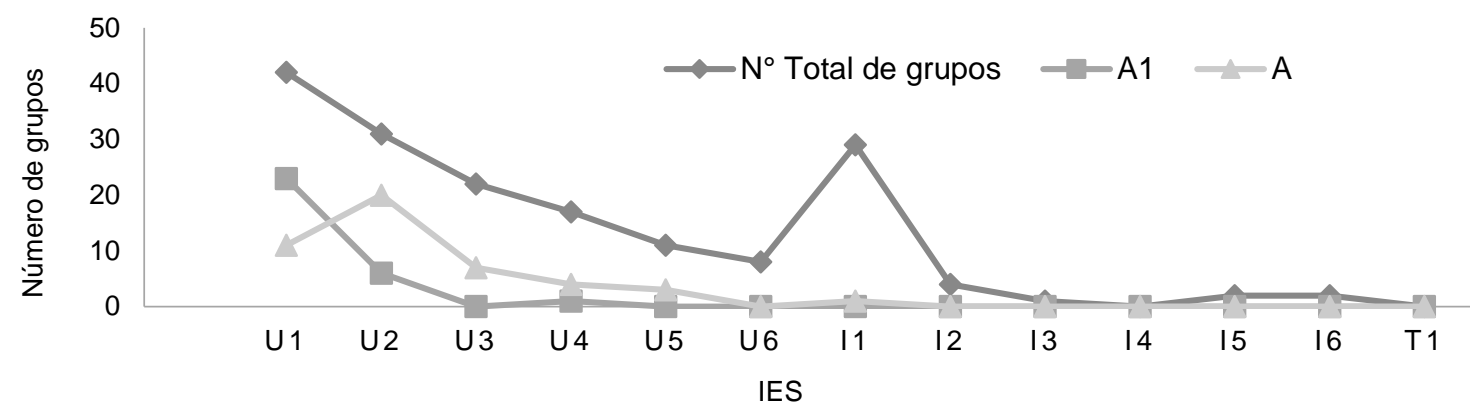

Fig. 5. Categorización de grupos de investigación.

Por un lado, esta categorización la han logrado gracias a reporte de publicaciones en revistas científicas alta visibilidad indexadas en alguno de los índices bibliográficos de citaciones ISI - Web of Knowledge (Science citation Index ISI), Social Citation Index (SSCI) o Scopus o en revistas científicas indexadas en alguno de los índices bibliográficos que garanticen la calidad científica de la política editorial y por otra parte por sus desarrollos tecnológicos. En la figura 6., se observa como las IES con más grupos en la categoría A1 y A son las que con mayor producción científica cuentan.

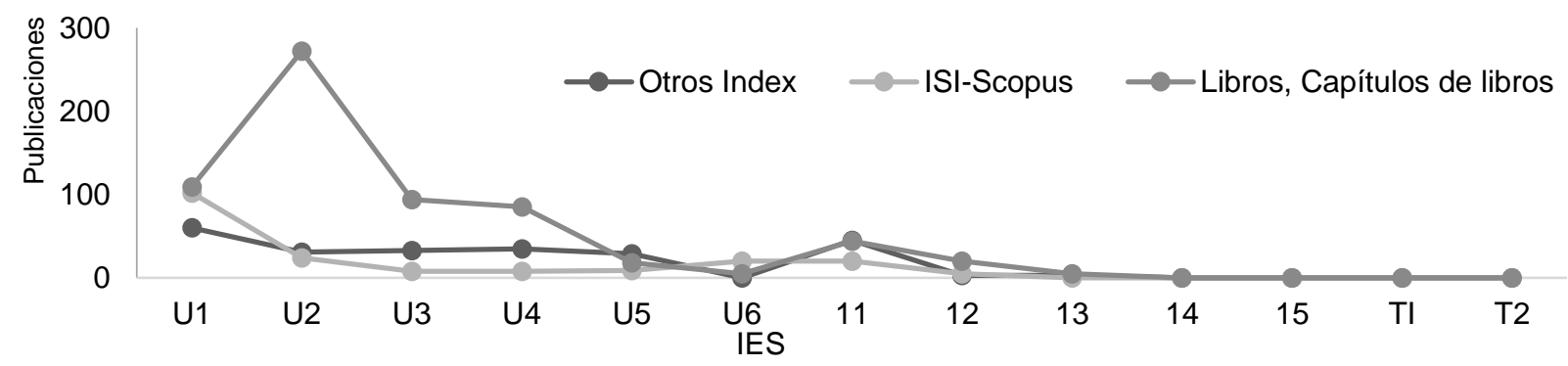

Fig. 6. Producción científica

En la figura 6, se evidencia que la situación es semejante cuando se revisan los proyectos que desarrollan las IES que cuentan con financiación externa, se observa que hay una tendencia a que la mayoría de los proyectos sean financiados con convenios o contratos provenientes del sector público para el beneficio del tejido empresarial y estos son ejecutados por las IES con mayor carácter académico.

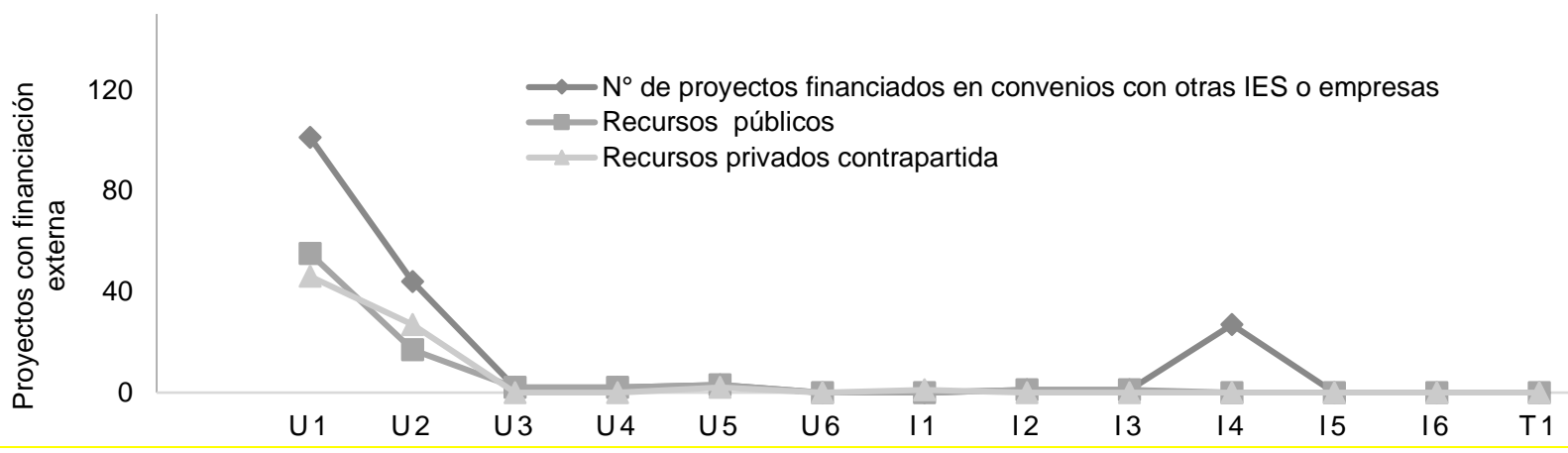

Fig. 7: Proyectos con financiación

En esta misma línea de análisis, a partir de la información reportada por las Instituciones de educación superior se analizaron las actividades de extensión y servicios externos, así como la interacción de las actividades identificadas con las funciones misionales de las IES. En la tabla 1 se describen las actividades 
de extensión y servicios externos realizadas. Por una parte, se evidenció que en su mayoría son las Universidades las que realizan convenios con el sector productivo y las que disponen de unidades de unidades de emprendimiento constituidas y tan solo dos de ellas cuentan laboratorios de innovación y emprendimiento y con observatorios de carácter científicos o académicos. El panorama es menos alentador respecto a las que tienen con departamentos de investigación constituidos, centros de asesoría, consultoría y asistencia técnica o consultorios jurídicos. Respecto a el análisis de los programas y actividades generales de articulación universitaria permite observar que todas las IES ofrecen programas de educación continua, pero son las Universidades las que cuentan con proyectos comunitarios de proyección social y dos de estas IES cuentan con reconocimientos dados por el sector empresarial.

En este sentido, el papel tradicional de las IES como generadora de graduandos se ve dinamizada al trasladar el conocimiento fuera de sí misma, mediante estrategias que le permiten la interacción entre sus actividades internas de docencia e investigación, con instituciones del medio externo con las que puede generar beneficios mutuos. Es así como se evidenciaron varios casos de éxitos de articulación Universidad- EmpresaEstado que generan capacidad de innovación, contribuyendo a la transformación de vidas dentro y fuera del escenario académico. Unos de estos casos se describe en lo que sigue.

Tabla 1. Actividades de extensión y servicios con externos (Adaptada de Barrios y Olivero, 2015)

\begin{tabular}{|c|c|c|c|c|c|c|c|c|c|c|c|c|c|c|c|}
\hline & \multicolumn{15}{|c|}{ Actividades } \\
\hline IES & 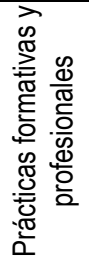 & 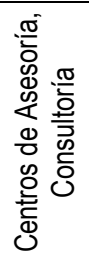 & 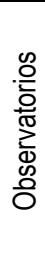 & 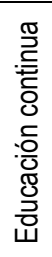 & 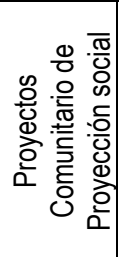 & 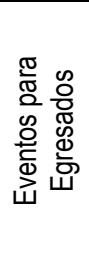 & 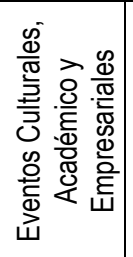 & 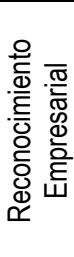 & 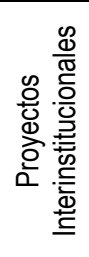 & 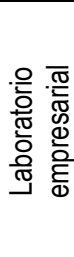 & 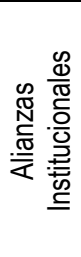 & 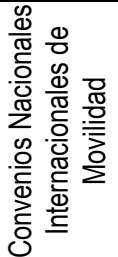 & 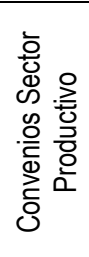 & 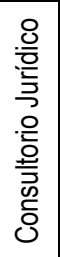 & 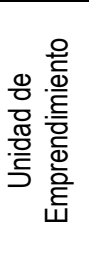 \\
\hline U1 & $x$ & $x$ & $x$ & $x$ & $x$ & $x$ & $x$ & $x$ & $x$ & $x$ & $x$ & $x$ & $x$ & $\mathrm{x}$ & $x$ \\
\hline $\mathrm{U} 2$ & $x$ & $x$ & & $\mathrm{X}$ & $x$ & $x$ & $x$ & $x$ & & $\mathrm{X}$ & $x$ & $x$ & $x$ & $x$ & $x$ \\
\hline U3 & & $x$ & $x$ & $x$ & $x$ & & $x$ & & $x$ & & $x$ & $x$ & & & $x$ \\
\hline U4 & & & & $x$ & $x$ & & & & & & & $x$ & $x$ & & $x$ \\
\hline U5 & & & & $\mathrm{X}$ & $X$ & & & & & & & & & & \\
\hline U6 & $\mathrm{X}$ & & & $\mathrm{X}$ & & & & & & & & $x$ & $x$ & & \\
\hline 11 & $x$ & & & $x$ & & & & & & & & $x$ & & & \\
\hline 12 & & & & $x$ & & & & & & & & & & $\mathrm{x}$ & $x$ \\
\hline 13 & & & & $\mathrm{X}$ & & & & & & & & $\mathrm{X}$ & $X$ & & \\
\hline 14 & & & & $x$ & & & & & & & & & & & \\
\hline 15 & & & & $x$ & & & & & & & & $x$ & & & \\
\hline 16 & & & & $x$ & $x$ & & & & & & & $x$ & & & \\
\hline $\mathrm{TI}$ & $x$ & & & $x$ & & & & & & & & $x$ & & & \\
\hline
\end{tabular}

\section{El caso del Centro de Crecimiento Empresarial MacondoLab}

Este centro nace en el año 2014 en la Universidad Simón Bolívar, integrando cuatro componentes fundamentales: la capacidad existente, la decisión de la alta dirección, las tendencias globales, y las oportunidades que había en el sector empresarial. Actualmente trabaja con un modelo diferenciador que les permite desarrollar soluciones empresariales basadas en el conocimiento. Este ha sido validado a nivel nacional e internacional por el sector empresarial, público y la academia. Adicionalmente, cuentan con un talento humano de alto nivel y experiencia. Se caracterizan porque son ágiles, flexibles y cálidos Han sido galardonados como Top Challenge Incubator por UBI y reconocidos como la primera aceleradora de Colombia y Top 13 de América Latina por Gust- Fundacity (MacondoLab, 2019). En el 2018 fue catalogada como la única aceleradora empresarial del Caribe colombiano en temas de industria creativa y cultural, por el Banco Interamericano de Desarrollo (BID). El 2019 obtuvo una nueva distinción por parte de la compañía y comunidad de inteligencia sueca UBI Global, que por segundo año consecutivo la reconoció entre las mejores del mundo en la categoría 'Top - Challenger Latinoamérica' por su valor para las nuevas empresas de clientes y el ecosistema local (El Tiempo, 2019). En MacondoLab se generan capacidades investigativas e innovadoras en empresas, mediante procesos de co-creación y desarrollos tecnológicos para la transformación del conocimiento en resultados a corto plazo. MacondoLab trabaja en articulación con el estado, a través de las Alcaldías, Gobernaciones, Ministerios, etc, el sector empresarial y otras IES. Ha desarrollado un sin número de proyectos que le ha permitido convertirse en un referente en el ecosistema empresarial del país (MacondoLab, 2019). 
Tal es el caso del programa Probeta, el cual, a través de la relación entre la Alcaldía Distrital de Barranquilla, la Academia y el sector empresarial, fortalece las empresas que basan sus desarrollos tecnológicos en investigación. De esta manera, se implementan estrategias para el fomento y desarrollo de modelos de negocios tecnológicos de alto impacto, a través de la articulación y potencialización de capacidades, la colaboración y el aprendizaje orientado al desarrollo y uso de tecnologías como apuestas que impulsen la competitividad y la innovación de la ciudad de Barranquilla. Probeta busca promover el desarrollo de capacidades científicas, tecnológicas y de innovación de empresas en sectores estratégicos de alto impacto en el Distrito de Barranquilla. Beneficiando desde su creación 23 empresas basadas en procesos de investigación, que generaron desarrollo de productos innovadores (MacondoLab, 2019)

Por otra parte, también se analizó la Unidad de Apoyo al Empresario como la estrategia de articulación entre la Alcaldía Distrital de Barranquilla y la Universidad Simón Bolívar para el fortalecimiento de las habilidades organizacionales de los emprendedores y empresarios de la ciudad. Nace en el 2016 con un enfoque de formación a microempresarios, para evitar o disminuir el endeudamiento innecesario de estos negocios. De este proceso se desprende el libro resultado de investigación "Unidad de Apoyo al Microempresario: hacia la construcción de microempresas sostenibles en Barranquilla", donde se muestran los impactos de la estrategia de política pública de la Alcaldía Distrital de Barranquilla, encaminada al fortalecimiento empresarial en sus diferentes sectores. En el 2017 la Unidad de Apoyo al Microempresario pasa a denominarse Unidad de Apoyo al Empresario, ampliando el nicho de atención. Los servicios se centraron en: capacitación en barrios, formación en temas financieros, mercadeo y ventas, finanzas, proyecto de vida y aspectos legales y asesorías. Adicionalmente se creó el programa Quilla Innova con la intención de acompañar a las empresas en el diseño y validación de modelos de negocios en etapa de ideación. En el 2018 se ofertaron talleres específicos para emprendedores y empresarios y Quilla Innova pasó a ser un programa de consolidación empresarial, dirigido a emprendedores y empresarios con un producto mínimo viable. Gracias al cúmulo de experiencia generada en estos años, el 2019 se enmarca en cuatro servicios principales: a) formación: talleres especializados y capacitación en barrios; b) asesorías especializadas; c) Quilla Innova, d) Quilla emprende, que nace como una ruta emprendedora dirigida a emprendedores o empresarios en etapa inicial.

\section{CONCLUSIONES}

De acuerdo al trabajo presentado y a los resultados obtenidos, se pueden plantear las siguientes conclusiones principales:

a) Las IES en Barranquilla son organizaciones que responden a través de sus procesos misionales de docencia, investigación y extensión a los cambios del entorno, generando con sus acciones sostenibilidad en el mercado, sin que ello signifique una amenaza para su misión académica tradicional (Clark, 1998; 2004; Barrios, Olivero, y Acosta-Prado, 2017). Siendo estas importante para el desarrollo del país, se hace recomendable afianzar la relación Universidad- Empresa- Estado para el desarrollo de capacidad de innovación con miras detectar oportunidades y amenazas (Teece, 2009), para la construcción de entornos colaborativos (Roncancio, 2011). Esto depende en gran medida de las alianzas y convenios con el sector productivo, que unidas a las relaciones con el gobierno, a través de sus diferentes entes reguladores, contribuyan a la creación de estos entornos.

b) Con base en los resultados encontrados es posible argumentar que la evidencia mostrada por Etzkowitz y Leydesdorff (2000) es acertada en cuanto las relaciones de la triple Hélice son un componente clave en la estrategia de la innovación tanto a nivel nacional como multinacional. Por otra parte, las características de cada una de las IES, permite reflexionar que entre mayor es el carácter académico y la experiencia de las mismas, mayor es la interacción a través de las relaciones con el estado y las empresas y por ende aumenta su participación en la dinámica del tejido empresarial y su reconocimiento en el ecosistema de la innovación.

c) La relación Universidad- Empresa- Estado contribuye en el desarrollo de capacidad de innovación en la medida que las IES dispongan de la habilidad para convertir la información y el know-how en productos y servicios comercialmente viables (Ghafele, 2012), porque en una sociedad que cambia constantemente, la innovación es una necesidad de la institución, no solo para su supervivencia sino para garantizar su sostenibilidad, siempre y cuando ésta permita la reconfiguración de la base de recursos y la adaptación a las condiciones cambiantes del mercado, con el objetivo de lograr una ventaja competitiva (Zahra y George, 2002; (Barrios, Olivero y Acosta-Prado, 2017)

\section{REFERENCIAS}

Acosta, J. y Fischer, A. Condiciones de la gestión del conocimiento, capacidad de innovación y resultados empresariales. Un modelo explicativo. Pensamiento y Gestión, Nº 35 (2013).

Barrios, K. y Olivero, E. La Innovación en Instituciones de Educación Superior Un Modelo Basado en Capacidades Dinámicas. Barranquilla: Universidad Simón Bolívar (2015).

Barrios, K., Olivero, E. y Acosta-Prado, J. Capacidad dinámica de innovación en instituciones de educación superior. Revista Espacios. Vol 38 (1), 24 (2017) 
Barrios-Hernández, K., Olivero Vega, E y otros veintitrés autores. Gestión del conocimiento y capacidad de innovación. Modelos, Sistemas y Aplicaciones. Barranquilla: Universidad Simón Bolívar (2017).

Blanco-Ariza, A., Rodríguez-Calderón, G y otros trece autores. Programa de Articulación de las Funciones de Docencia, Investigación, Extensión y Proyección Social (PADIE). Un enfoque desde la Facultad de Administración y Negocios. Barranquilla, Colombia: Ediciones Universidad Simón Bolívar (2017).

Bueno, E. La tercera misión de la Universidad: el reto de la trasferencia del conocimiento. Revista Electrónica de Madrid, 41, 11, marzo-abril (2007)

Castellanos, O., Chávez, R y Jiménez, C. Propuesta de formación en liderazgo y emprendimiento. Innovar, Revista de Ciencias Administrativas y Sociales 22, 145-156 (2003).

Clark, B. Creating Entrepreneurial Universities: Organizational Pathways of Transformation. Oxford: Pergamon-Elsevier (1998).

Clark, B. Sustaining Change in Universities, Society for Research into Higher Education. Londres: Open University Press (2004).

Cohen, W. y Levinthal, D. Absorptive Capacity: A new perspective on learning and inn0ovation. Adminsitrative Science Quaterly, Vol 35(1), 128-152 (1990).

Dávila, J. Capacidades organizacionales: dinámica por naturaleza. Cuadernos de Administración 26(47), 11-33 (2013).

El Tiempo. Más de 55 mil emprendedores han sido impactados por MacondoLab. Recuperado de https://www.eltiempo.com/contenido-comercial/mas-de-55-mil-emprendedores-han-sido-impactados-por-macondolab444236. (2019)

Etzkowitz, H., y Leydesdorff, L. The Triple Helix University Industry Gobernment Relations: A laboratory for Knowledge based Economic Development. EASST Review (14), 14-19 (1995).

Etzkowitz, H. y Leydesdorff, L. The Dynamic of Innovation: from National Systems and "mode 2" to triple Helix of UniversityIndustry- Government Relations. Elsevier Science, 109-123 (2000).

Garzón, M. El Constructo capacidades dinámicas. Guanajuato, México: Ide@s CONCYTEG (8) 99. (2013).

Ghafele, R. Financing University Research. University of Oxford, Oxfirst. (2012)

Gros, B., y Lara, P. Estrategias de innovación en la educación superior: El caso de Universitat Oberta de Catalunya. Revista Iberoamericana de Educación (49), 223-245 (2009).

Kogut, B., y Zander, U. Knowledge of firma, combinative capabilities and the replication of technology. Organization Science Vol 3(3), 383-397(1992).

Lane, P. J., Koka, B. R. y Pathak, S. "The reification of absorptive capacity: a critical review and rejuvenation of the construct". https://doi.org/10.5465/amr.2006.22527456. Academy of Management Review, 31, 833-863 (2006).

Lundvall, B. National Systems and National Styles of Innovation. Fourth. Fourth International ASEAT Conference "Differences in 'styles'. Manchester (1997).

MacondoLab. Términos de referencia Probeta 3.0. (2019)

MacondoLab. www. Macondolab.com. Recuperado el 2019, de https://macondolab.com/nosotros (2019)

Morales. M., Pineda, K. y Avila, K. Organizaciones innovadoras a partir de la interacción con la universidad: casos exitosos. Estudios gerenciales, 363-374 (2012)

Roncancio, P. De las capacidades dinámicas como enfoque de la estrategia a la integración de competencias para la construcción de un entorno colaborativo universidad - empresa. Revista Ciencias Estratégicas. Vol. 19 (26), 295-305 (2011).

Talaya, Á., y Collado, A. Investigación de Mercados, 1a Ed., 8-209, Esic Editorial, Madrid, España (2014)

Venture, V. Análisis competitivo de la empresa: un enfoque estratégico. Civitas: Madrid (1994).

Villaveces, J. Nuevas políticas de ciencia y tecnología. En: H. Vessuri. Universidad e investigación científica., 193-205 (2006).

Vivas, S. Implicaciones de las capacidades dinámicas para la competitividad y la innovación en el siglo XXI.,. Cuadernos de Administración, 26 (47), 119-139 (2013).

Wang, C., y Ahmed, P. Dynamic Capabilities: "A review and Research Agenda". Stretegic Management Journal, 31-51 (2007).

Winter, S. Understanding dynamic capabilties. https://doi.org/10.1002/smj.318. Strategic Management Review (24), 991995 (2003).

Zahra, S., y George, G. Absorptive capacity: A review, reconceptualisation, and extension. Academy of Management https://doi.org/10.2307/4134351. Review 27(2), 185-203 (2002). 